\title{
Research on Optimal Location of Logistics Distribution Center Based on the Improved Gray Relation
}

\author{
Shuguo Zhang ${ }^{\mathrm{a}}$, Qian $\mathrm{Li}^{\mathrm{b}, \text { * }}$ \\ Department of Economic Management, North China Electric Power University, Baoding, 071003, \\ China \\ a email: 18932660801@163.com, ${ }^{\mathrm{b} e m a i l: ~ c h e e r u p g o w e l l @ 163 . c o m, ~}{ }^{*}$ corresponding author
}

Keywords: Gray relational analysis, Entropy weight method, Euclidean distance, Manhattan distance

\begin{abstract}
Based on the analysis of the existing logistics and distribution center location methods, this paper builds the evaluation index system of logistics and distribution center selection and establishes the gray relational location module as the model. It applies the improved entropy method to the evaluation index and the Euclidean distance is introduced. The optimal gray relational analysis method is used to obtain the optimal site. This paper is in combination with analysis and the results between the Euclidean distance and the Manhattan distance which shows that the method is effective and it improves the location efficiency. It is also suitable for practicing in other aspects of the location problem.
\end{abstract}

\section{Introduction}

With the rapid development of world economy and technology, logistics distribution as a new service is now developing rapidly in the world. An advanced logistics distribution system not only speeds up economic growth, but also reduces unnecessary transaction costs. The issues of logistics distribution center location include how to choose the location of the potential distribution center and how to transport the product through the distribution center. In regard to the research of logistics distribution center location, there are many classic algorithms to solve such problems. Take these for example: Shiqiang Xu (2014) [1] used the combination weighting method and used TOPSIS method to establish the logistics center location evaluation model. Rong Yu et al., (2015) [2] used AHP to determine the weight of indicators at all levels, and built the fuzzy comprehensive evaluation model. These methods of research have some problems to different degrees. In this paper, the traditional entropy method is improved and the malpractice is overcome. The scope of its use is expanded to empower the index. Finally, the gray relational analysis method is used to evaluate the logistics distribution center address comprehensively.

\section{The Basic Idea of Modeling}

This paper mainly uses the gray relocation site module: First of all, this paper selects the factors which affect the location of the logistics distribution center and establishes the evaluation index system. Secondly, the model is established and it is analyzed by the improved gray relational analysis method to calculate the relative value of the logistics distribution center. Finally, it determines the optimal site and uses data to analyze sample points to determine the best choice for site selection and draw conclusions [3].

\section{Construction of Evaluation Index System}

Infrastructure improvement: Infrastructure improvement refers to the construction circumstances of the road traffic conditions about distribution center and electricity, water and other public facilities. Perfect infrastructure effectively protects the normal operation and operation of logistics distribution center.

Economic factors: Economic factors mainly include the rents of local land, logistics warehousing 
construction costs and local labor costs. Costs should be considered in logistics distribution center location issues to process, package and distribute goods well.

Natural environment: The natural environment mainly refers to the local meteorological conditions, geological conditions, hydrologic conditions and terrain conditions. In terms of meteorological conditions, the main considerations of meteorological conditions are temperature, wind and other indicators. Geological conditions require that the soil of the logistics distribution center should possess sufficient capacity. Hydrologic conditions require that logistics distribution centers should be far away from easily flooded areas. Terrain conditions require site selection should choose higher and flat terrain.

Government support: Logistics distribution center should also consider the local government support. The greater support of the local government provides, the more suitable the establishment of distribution center is.

Ecological benefits: Ecological benefits mainly refer to the ecological environment which is near the selected center and it should consider whether it has a sustainable development trend [4].

\section{Logistics Distribution Center Location Step Based on the Improved Gray Relational Analysis Method and the Improved Entropy Weight Method}

This paper supposes that in the evaluation system of commercial network location ,there are $\mathrm{m}$ evaluation samples $A_{i}, i \in M=\{1,2, \ldots, m\}$ and $n$ evaluation indexes $E_{j}, j \in N=\{1,2, \ldots ., n\}$. Let the evaluation matrix be $Y=\left(Y_{i j}\right)_{m^{*} n}$ and $y_{\mathrm{ij}}$ is the attribute value of the $i$-th evaluation sample under the $j$-th index. The gray relational analysis procedure is as follows:

(1) Use the range-method to standardize the original data processing and set the standard matrix as the $X=\left(X_{i j}\right)_{m * n}$.

For positive indicators:

$$
x_{i j}=\frac{y_{i j}-\min \left\{y_{i j}\right\}}{\max \left\{y_{i j}\right\}-\min \left\{y_{i j}\right\}}, 1 \leq i \leq m, 1 \leq j \leq n
$$

For negative indicators:

$$
x_{i j}=\frac{\max \left\{y_{i j}\right\}-y_{i j}}{\max \left\{y_{i j}\right\}-\min \left\{y_{i j}\right\}}, 1 \leq i \leq m, 1 \leq j \leq n
$$

(2) Use the improved entropy weight method to determine the weight:

In order to overcome the limitations of the special conventions of the entropy method, it is not necessary to assume that the special scope of $r_{i j}=0, r_{i j} \ln \left(r_{i j}\right)=0$. It expands its scope of application and makes it easier to use. The specific calculation steps are as follows [5]:

1) Method of determining the entropy about each index:

$$
P_{i j}=-K \sum_{i=1}^{m} r_{i j} \ln \left(r_{i j}\right)
$$

In the formula:

${ }^{{ }^{1}} r_{i j}=\frac{Y_{i j}+1}{\sum_{i=1}^{m}\left(Y_{i j}+1\right)},\left(K=\frac{1}{\ln (m)}, j=1,2, \ldots, n\right)$

2) Method of determining the difference coefficient about each index:

$H_{j}=1-P_{j}$

3) Method of determining weight

Standardize index difference coefficient and you can get the weight. The formula is:

$$
\omega_{j}=\frac{H_{j}}{\sum_{j=1}^{n} H_{j}}
$$

(3)Calculate the weighted normalization decision matrix:

$$
Z=\left(z_{i j}\right)_{m * n}
$$

In the formula: $z_{i j}=\omega^{*} x_{i j}, \quad i \in M, j \in N$ 
(4) Determine the positive ideal solution $z^{+}$and the negative ideal solution $z^{-}$of the weighted normalized matrix $Z$ :

$$
Z^{+}=\left(z_{1}^{+}, z_{2}^{+}, \ldots, z_{n}^{+}\right), Z^{-}=\left(z_{1}^{-}, z_{2}^{-}, \ldots, z_{n}^{-}\right)
$$

In the formula: $z_{j}^{+}=\max _{i}\left\{z_{i j}\right\}=\omega_{j}, z_{j}^{-}=\min _{i}\left\{z_{i j}\right\}=0$

(5) Calculate the gray correlation coefficient matrix of each evaluation sample and positive and negative ideal solution:

$$
\begin{aligned}
& R^{+}=\left(r_{i j}^{+}\right)_{m *_{n}} \text { andR } R^{-}=\left(r_{i j}^{-}\right)_{m * n} \\
& r_{i j}^{+}=\frac{\min \left|z_{j}^{+}-z_{i j}\right|+\xi \max \left|z_{j}^{+}-z_{i j}\right|}{\left|z_{j}^{+}-z_{i j}\right|+\xi \max \left|z_{j}^{+}-z_{i j}\right|}=\frac{\xi \omega_{j}}{\omega_{j}-z_{i j}+\xi \omega_{j}} \\
& r_{i j}^{-}=\frac{\min \left|z_{j}^{-}-z_{i j}\right|+\xi \max \left|z_{j}^{-}-z_{i j}\right|}{\left|z_{j}^{-}-z_{i j}\right|+\xi \max \left|z_{j}^{-}-z_{i j}\right|}=\frac{\xi \omega_{j}}{z_{i j}+\xi \omega_{j}}
\end{aligned}
$$

In the conclusion, $\xi$ is identification coefficient $(0<\xi<1)$. When $\xi \leq 0.5463$, resolution is best and $\xi$ is 0.5 generally.

(6) Calculate the gray correlation degrees among each sample and positive and negative ideal solution:

$$
r_{i}^{+}=\frac{1}{n} \sum_{j=1}^{n} r_{i j}^{+}, r_{i}^{-}=\frac{1}{n} \sum_{j=1}^{n} r_{i j}^{-}
$$

(7) Calculate the Euclidean distance $d_{i}{ }^{+}$and $d_{i}{ }^{-}$and the Manhattan distance $d_{i}{ }^{+{ }^{*}}$ and $d_{i}{ }^{-{ }^{*}}$ which are from the evaluation sample to the positive and negative ideal solution:

$$
d_{i}^{+^{+}}=\sum_{j=1}^{n}\left|z_{i j}-z_{j}^{+}\right|, d_{i}^{-}=\sum_{j=1}^{n}\left|z_{i j}-z_{j}^{-}\right| d_{i}^{+}=\sqrt{\sum_{j=1}^{n}\left(z_{i j}-z_{j}^{+}\right)^{2}}, d_{i}^{-}=\sqrt{\sum_{j=1}^{n}\left(z_{i j}-z_{j}^{-}\right)^{2}}
$$

(8) Make the degree of correlation, Euclidean distance and Manhattan distance being dimensionless respectively:

$$
R_{i}^{+}=\frac{r_{i}^{+}}{\max r_{i}^{+}}, R_{i}^{-}=\frac{r_{i}^{-}}{\max r_{i}^{-}}, D_{i}^{+}=\frac{d_{i}^{+}}{\max d_{i}^{+}}, D_{i}^{-}=\frac{d_{i}^{-}}{\max d_{i}^{-}}, D_{i}^{+^{-}}=\frac{d_{i}^{+}}{\max d_{i}^{+}}, D_{i}^{-}=\frac{d_{i}^{-}}{\max d_{i}^{-}}
$$

(9)Calculate the comprehensive correlation degree. Set as follows:

$$
S_{i}^{+}=\alpha R_{i}^{+}+\beta D_{i}^{-}, S_{i}^{-}=\alpha R_{i}^{-}+\beta D_{i}^{-} \text {, and } i \in M
$$

In the formula, $\alpha$ and $\beta$ reflect the degree of preference about the position and shape of the decision maker. This formula satisfies $\alpha+\beta=1$, and $\alpha, \beta \in[0,1]$. Decision maker can determine the value according to their own preferences, generally $\alpha=\beta=0.5 . S_{i}^{+}$reflects the degree of closeness which is about the evaluation sample and the ideal solution. The larger the value is, the better the sample is. $S_{i}{ }^{-}$reflects the degree of closeness which is about the evaluation unit and the ideal solution. The larger the value is, the worse the sample is.

(10) Calculate the relative proximity of the sample:

$$
C_{i}^{+}=\frac{S_{i}^{+}}{S_{i}^{+}+S_{i}^{-}} \text {and } i \in M \text {. }
$$

The greater the degree of closeness is, the better the sample is. The smaller the degree of closeness is, the worse the sample is.

\section{Case study}

In order to expand the domestic product supply market, Cai Rentian Packaging Co., Ltd. is preparing for a product logistics distribution center in Canglong Island area. After the previous market research and inspection, the final selections of five candidates address are $A_{1}, A_{2}, A_{3}, A_{4}, A_{5}$. The expert groups evaluate the candidate addresses from the five aspects: infrastructure improvement, economic impact factors, natural environment, government support and ecological benefits. The 
evaluation values are given in the form of full point as 1 point. The second evaluation indicator is a cost indicator. In the process of processing the evaluation data, use 1 point to subtract the corresponding evaluation value to make all evaluation indexes be benefit type indicators. After analysis, we can see that economic factor is negative indicator and the others are positive indicators. In that case, it is necessary to process the data from the expert evaluation. Obtain the following evaluation matrix:

Table 1 Evaluation Data of Logistics Distribution Center Alternative Address

\begin{tabular}{|c|c|c|c|c|c|}
\hline Sample / indicator & $C_{1}$ & $C_{2}$ & $C_{3}$ & $C_{4}$ & $C_{5}$ \\
\hline$A_{1}$ & 0.8147 & 0.7785 & 0.9572 & 0.7922 & 0.6787 \\
\hline$A_{2}$ & 0.9058 & 0.5469 & 0.9854 & 0.9595 & 0.7577 \\
\hline$A_{3}$ & 0.6270 & 0.9575 & 0.8003 & 0.6557 & 0.7431 \\
\hline$A_{4}$ & 0.9134 & 0.9649 & 0.6419 & 0.5357 & 0.8922 \\
\hline$A_{5}$ & 0.6324 & 0.6576 & 0.9218 & 0.8491 & 0.6555 \\
\hline
\end{tabular}

(1) Calculate the standard and weight, as shown in Table 2

Table 2 Standard values and weights for evaluation indicators

\begin{tabular}{|c|c|c|c|c|c|}
\hline Sample / indicator & $C_{1}$ & $C_{2}$ & $C_{3}$ & $C_{4}$ & $C_{5}$ \\
\hline$A_{1}$ & 0.6554 & 0.4459 & 0.9179 & 0.6052 & 0.0980 \\
\hline$A_{2}$ & 0.9735 & 1.0000 & 1.0000 & 1.0000 & 0.4318 \\
\hline$A_{3}$ & 0.0000 & 0.0177 & 0.4611 & 0.2832 & 0.3701 \\
\hline$A_{4}$ & 1.0000 & 0.0000 & 0.0000 & 0.0000 & 1.0000 \\
\hline$A_{5}$ & 0.0189 & 0.7352 & 0.8148 & 0.7395 & 0.0000 \\
\hline Improved entropy weighting & 0.2955 & 0.2223 & 0.1904 & 0.1576 & 0.1341 \\
\hline
\end{tabular}

Calculate the relative proximity according to the improved gray relational analysis procedure:

(2) Calculate the weighted normalization decision matrix
$\mathrm{Z}=$
0.1937
0.0991
0.1748
0.0954
0.0131
$0.2877 \quad 0.2223$
0.1904
0.1576
0.0579
$0.0000 \quad 0.0039$
0.0878
0.0446
0.0496
0.2955
0.0000
0.0000
0.0000
0.1341
0.0056
0.1634
0.1551
0.1165
0.0000

(3) Calculate the gray correlation coefficient matrix of each sample and positive and negative ideal solution

$\mathrm{R}^{+}=\left(\begin{array}{lllll}0.5921 & 0.4755 & 0.8592 & 0.5589 & 0.3566 \\ 0.9449 & 1.0000 & 1.0000 & 1.0000 & 0.4681 \\ 0.3333 & 0.3373 & 0.4813 & 0.4108 & 0.4424 \\ 1.0000 & 0.3333 & 0.3333 & 0.3333 & 1.0000 \\ 0.3376 & 0.6536 & 0.7295 & 0.6572 & 0.3333 \\ & & & & \\ 0.4327 & 0.5287 & 0.3526 & 0.4524 & 0.8366 \\ 0.3393 & 0.3333 & 0.3333 & 0.3333 & 0.5366 \\ 1.0000 & 0.9661 & 0.5202 & 0.6386 & 0.5748 \\ 0.3333 & 1.0000 & 1.0000 & 1.0000 & 0.3333 \\ 0.9635 & 0.4048 & 0.3803 & 0.4035 & 1.0000\end{array}\right.$

(4) Make the degree of correlation and Euclidean distance being dimensionless

$R_{i}^{+}=(0.6434,1.0000,0.4538,0.6790,0.6136)$

$R_{i}^{-}=(0.7036,0.5071,1.0000,0.9911,0.8520)$

$D_{i}^{+}=(0.5176,0.1883,1.0000,0.8173,0.8097)$

$D_{i}^{-}=(0.6658,1.0000,0.2488,0.7318,0.5722)$

(5) Calculate the comprehensive correlation degree (Euclidean distance)

$S_{i}^{+}=(0.6546,1.0000,0.3513,0.7054,0.5929)$

$S_{i}^{-}=(0.6106,0.3477,1.0000,0.9042,0.8309)$ 
(6) Calculate the relative proximity of the sample (Euclidean distance)

$C_{i}^{+}=(0.5174,0.7420,0.2600,0.4382,0.4164)$

From the calculation results, the maximum value which uses the improved algorithm and the Euclidean distance is 0.7420 , that is, the sample 2 is the best point and the sample 1 is the secondary one.

Similarly, we can get the results of Manhattan distance: $C_{i}^{+^{+}}=(0.5049,0.7662,0.2451,0.4005,0.4156)$.

Manhattan distance is not a distance invariant. Manhattan distance diagram first came into being in the early computer graphics. The screen is composed of pixels and it is the integer. The coordinates of the point is generally an integer because the floating-point operation is very expensive, very slow and error. If we directly use AB's Euclidean distance (Euclidean distance: in the two-dimensional and three-dimensional space, it is the distance between the two points), we must carry out floating-point operations, greatly improving the speed of operation. No matter how many times the cumulative operation, there will be no error.

From the calculation results, the maximum value which uses the improved algorithm and Manhattan distance is 0.7662 , that is, sample 2 is the best point, sample 1 is the secondary one.

In conclusion, optimal location results are the same when we use the Euclidean distance formula and the Manhattan distance formula: the sample 2 is the best and the sample 1 is the secondary one.

\section{Conclusion}

In this paper, we introduce the Euclidean distance and the improved entropy method to improve the traditional gray relational analysis method. Through the establishment of the gray relational location module and the improvement of the original entropy weight method, we can expand its scope. This paper analyzes the evaluation index system of the logistics distribution center from five aspects. Finally, in combination with the case study, the Euclidean distance is compared with the Manhattan distance to find optimal location and it has a reference to the logistics and distribution center site.

\section{References}

[1]Shiqiang Xu. Research on Logistics Center Location Based on Combination Weighted TOPSIS Model[J]. Logistics Technology,2014,11:132-134.

[2] Rong Yu, Jiafeng Chen, Ya Gong, Bin Zhou. Study on the Location of Logistics Distribution Center Based on Fuzzy Comprehensive Evaluation Method - A Case Study of Debong Logistics in Taixing City, Jiangsu Province[J]. Science and Technology Management of Land and Resources,2015,04:93-100.

[3] Qingxiang Zhu, Qiufang Cao, Jing Liu, Haipeng Ji, Zhipeng Wang, Siyuan Lei. Research on Optimal Location of Commercial Network Based on Improved Gray Relation[J]. Statistics and Decision,2016,08:51-53.

[4] Zhanbo Chen, Xiaozhou Huang. An Improved Gray Relational Grade Evaluation Method for Logistics Distribution Center Location[J]. Statistics and Decision,2015,03:52-55.

[5] Baoquan Ning, Zhenpei Shan. Research on Logistics Distribution Center Location Based on Improved Gray Relational Model[J]. Journal of Liupan Shui Teachers College,2016,03:1-4. 